\title{
A New Local Approach for Flooding Level Estimation in Urban Areas Using Single SAR Images
}

\author{
Vincenzo Diessa, Pasquale Iervolino, Antonio Iodice, \\ Antonio Ricciardi, Daniele Riccio \\ DIBET \\ Università Federico II \\ Napoli, Italy \\ \{iodice, daniele.riccio\}@unina.it \\ \{v.diessa, p.iervolino, a.ricciardi\}@studenti.unina.it
}

\author{
Raffaella Guida \\ Surrey Space Centre \\ University of Surrey \\ Guildford, U.K. \\ R.Guida@surrey.ac.uk
}

\begin{abstract}
The aim of this paper is to evaluate the level of flooding in proximity of sensible targets in urban areas using only one Synthetic Aperture Radar (SAR) image. To this purpose a two-step algorithm is here proposed: first the flooded areas are detected in the SAR image; and then the water level is retrieved by inverting scattering models developed for urban areas and now properly adapted for the case at issue. The retrieval is performed through a local approach where the a-priori knowledge of the target ground truth and two gauges in the premises is required. The approach is tested on a High Resolution (HR) TerraSAR-X image acquired during the flooding occurred in the Gloucestershire in July 2007.
\end{abstract}

\section{INTRODUCTION}

Natural disasters are one of the major hazards affecting urban areas. A reliable and immediate response to crisis conditions is a necessary task. Moreover, in case of disasters like flooding, the environment is often greatly perturbed by atmospheric phenomena. For instance, it is highly likely that the presence of clouds makes the optical analysis of a flooded area an impossible task. In this case, it is desirable to monitor the area by means of a sensor able to acquire information independently from weather conditions and daytime. In this framework, Synthetic Aperture Radar (SAR) systems have been successfully applied for flood mapping and monitoring applications. In addition, when a flooding occurs, the surface profile is modified at several scales. The water tends to cover the ground and change the macroscopic topography as well as the roughness properties of the scene. In this paper the electromagnetic model presented in [1] is adopted with proper changes to consider the presence of water in an urban area, possible changes to the relative building height, the surface roughness and the dielectric properties of the new scenario. Obviously all these parameters affect the electromagnetic scattering of a building. In order to evaluate the flooding level, an adaptation of the approach illustrated in [2] for building height retrieval is here suggested. For example, the presence of water modifies the mechanism of double reflection: from one hand the building height decreases thus leading to a decrease of the double reflection brightness; from the other hand the permittivity of water and wet terrain is higher than that of dry soil and this causes an increment of the backscattered signal.

The paper is organized as follows: in Section II we describe in detail the technique used to delineate the boundaries of the flooded areas and the local approach which allows the extraction of the flooding level based on the knowledge of the electromagnetic and geometric models of three buildings. In Section III, we present the features of the HR SAR image of the area under study and the electromagnetic and geometric parameters of the local target. Finally, in Section IV the measurement results are reported and future perspectives are briefly commented.

\section{THE APPROACH}

The most important novelty and peculiarity of our approach consists in retrieving the inundation level by using a single SAR image. First of all we carried out a mapping of the region of interest localizing the boundaries of the flooded areas. There are many different methods to delineate the flooding areas [3][4]; we use a simple yet effective approach since classification is not the primary aim of this research. This algorithm considers two threshold values to identify the flood and nonflood pixels. The thresholds are chosen according to the statistics of the flooded areas image; in particular the low and high level threshold values are set by the mean value intensity diminished or augmented, respectively, by the corresponding standard deviation. Since the backscattering from water and the shadow appear both dark in a SAR image, it is possible to keep the shadow pixels out of the areas classified as flooded by using an appropriate minimum population value: the clusters of pixels whose population is smaller than the minimum population value are not regarded as flooded areas even if their grey levels belong to the range between the low and the high threshold. This delimitation is useful to localize the flooded buildings and the local targets for which the estimation of the water level is required. It is possible to evaluate the building height by recognizing the single scattering contribution from the wall: this can be done via the Kirchhoff approach (KA) within the Physical optics (PO) or Geometrical optics (GO) 
approximations, according to the ground roughness [1]; alternatively the double scattering or the triple contributions in the SAR image can be employed. Since the single and triple scattering contributions are often mixed, it is preferable to rely upon the double reflection one. According to the rationale in [1] it is possible to associate the building height to the contribution of the double scattering mechanism to the radar cross section:

$$
\sigma^{0}=h \cdot f(\mathbf{p})
$$

where $\sigma^{0}$ is the incoherent radar cross section and $\mathbf{p}$ is a known parameters vector, $\mathbf{p}=\left(l, \sigma, L, \mathcal{E}_{w}, \mathcal{E}_{s}, \varphi, \vartheta\right), l$ is the building length, $\sigma$ and $L$ are the standard deviation and the correlation length, respectively, of the stochastic process representing the ground surface, $\varepsilon_{w}$ is the complex dielectric constant of the building wall, $\varepsilon_{s}$ is the complex dielectric constant of the soil surface, $\varphi$ is the angle between the sensor line of flight and the projection of the building wall to the ground, $\vartheta$ is the SAR look angle. The function $f(\mathbf{p})$ is given by [1]:

$$
\begin{aligned}
f(\mathbf{p})= & \left|S_{p q}\right|^{2} l \tan \vartheta \cos \varphi \exp \left(-4 k^{2} \sigma^{2} \cos ^{2} \vartheta\right) \\
& \sum_{m=1}^{\infty} \frac{(2 k \sigma \cos \vartheta)^{2 m}}{m !} \frac{k^{2} L^{2}}{4 m} \exp \left[-\frac{(2 k L \sin \varphi \sin \vartheta)^{2}}{4 m}\right]
\end{aligned}
$$

for the GO-PO approach; alternatively:

$$
f(\mathbf{p})=\frac{\left|S_{p q}\right|^{2} l \tan \vartheta \cos \varphi\left(1+\tan ^{2} \vartheta \sin ^{2} \varphi\right) \exp \left[-\frac{\tan ^{2} \vartheta \sin ^{2} \varphi}{2 \sigma^{2}\left(2 / L^{2}\right)}\right]}{8 \pi^{2} \sigma^{2}\left(2 / L^{2}\right) \cos ^{2} \vartheta}
$$

for the GO-GO solution [2]. In (2) and (3), $S_{p q}$ is the generic element of the scattering matrix with $p$ and $q$ standing for horizontal, $h$, or vertical, $v$, polarization, respectively [1]; $S_{p q}$, depends on $\varepsilon_{w}, \varepsilon_{s}, \varphi$ and $\vartheta ; k=2 \pi / \lambda$ is the radar signal wavenumber with $\lambda$ the working wavelength. In order to proper link the grey intensity levels to the radar cross section it is necessary to calibrate the image: one multiplicative and one additive constant must be included in (1); furthermore, in presence of water, the height $h$ in (1) is given by the difference between the building height and the height of the water level. In this way it is possible to write a three equations system which permits to estimate the inundation level employing two gauge buildings with different known heights and the building of interest (local target). Obviously, we assume that the other electromagnetic and geometric parameters that do not change with the change of the scenario are either a priori known or have been previously retrieved from non-flooded SAR image [2]. The same water level for the three buildings is assumed. This means that the gauge buildings need to be in the surroundings of the target building, an assumption that makes the approach local. The system to solve is:
$\left\{\begin{array}{l}\hat{\sigma}_{A}^{0}=c\left(h_{A}-h_{W}\right) \cdot f\left(\mathbf{p}_{\mathbf{A}}\right)+d \\ \hat{\sigma}_{B}^{0}=c\left(h_{B}-h_{W}\right) \cdot f\left(\mathbf{p}_{\mathbf{B}}\right)+d \\ \hat{\sigma}_{C}^{0}=c\left(h_{C}-h_{W}\right) \cdot f\left(\mathbf{p}_{\mathbf{C}}\right)+d\end{array}\right.$

where $\hat{\sigma}_{i}^{0}$ is the generic radar cross section relevant to the double reflection mechanism as evaluated in correspondence of the $i$-th building in the SAR image; $c$ and $d$ are the multiplicative and additive calibration constants, respectively; $h_{i}$ is the height of the $i$-th building and $h_{W}$ is the water level of the flooding. Solving (4) it is possible to obtain the solutions shown in (5):

$\left\{\begin{array}{l}h_{W}=\frac{h_{A}\left(\hat{\sigma}_{C}^{0}-\hat{\sigma}_{B}^{0}\right) \cdot f\left(\mathbf{p}_{\mathbf{A}}\right)+h_{B}\left(\hat{\sigma}_{A}^{0}-\hat{\sigma}_{C}^{0}\right) \cdot f\left(\mathbf{p}_{\mathbf{B}}\right)+h_{c}\left(\hat{\sigma}_{B}^{0}-\hat{\sigma}_{A}^{0}\right) \cdot f\left(\mathbf{p}_{\mathbf{C}}\right)}{\left(\hat{\sigma}_{C}^{0}-\hat{\sigma}_{B}^{0}\right) \cdot f\left(\mathbf{p}_{\mathbf{A}}\right)+\left(\hat{\sigma}_{A}^{0}-\hat{\sigma}_{C}^{0}\right) \cdot f\left(\mathbf{p}_{\mathbf{B}}\right)+\left(\hat{\sigma}_{B}^{0}-\hat{\sigma}_{A}^{0}\right) \cdot f\left(\mathbf{p}_{\mathbf{C}}\right)} \\ c=\frac{\left(\hat{\sigma}_{A}^{0}-\hat{\sigma}_{B}^{0}\right)}{\left(h_{A}-h_{W}\right) \cdot f\left(\mathbf{p}_{\mathbf{A}}\right)-\left(h_{B}-h_{W}\right) \cdot f\left(\mathbf{p}_{\mathbf{B}}\right)} \\ d=\hat{\sigma}_{B}^{0}-\frac{\left(\hat{\sigma}_{A}^{0}-\hat{\sigma}_{B}^{0}\right)\left(h_{B}-h_{W}\right) \cdot f\left(\mathbf{p}_{\mathbf{B}}\right)}{\left(h_{A}-h_{W}\right) \cdot f\left(\mathbf{p}_{\mathbf{A}}\right)-\left(h_{B}-h_{W}\right) \cdot f\left(\mathbf{p}_{\mathbf{B}}\right)}\end{array}\right.$

It is possible to generalize this approach if the ground of the inundated area is almost flat and therefore it is possible to define the same water level for all the buildings. We are working to develop also a global approach. In the next section the algorithm proposed for the local approach is applied to the case study.

\section{CASE STUDY AND DATA SET}

In July 2007, Gloucestershire (U.K.) experienced its worst flood on record; Tewkesbury was one of the most damaged towns lying at the confluence of two rivers: the Severn from northwest and the Avon from northeast. One TerraSAR-X Stripmap image, shown in Fig.1(a), was acquired on the area on the 25th July 2007 and is used in this study. At that time the satellite was still in its commissioning phase but the image shows a lot of details of the flooded urban areas. The look angle is $24^{\circ}$, the spatial resolution respectively $3.3 \mathrm{~m}$ and $1.2 \mathrm{~m}$ for the azimuth and the slant range, the polarization mode is $\mathrm{HH}$. This polarization provides a better discrimination between flooded and non-flooded areas [5]. First of all, a threshold segmentation has been applied to the data set. The aim of this kind of segmentation is to evaluate if the local targets are located in the flooded areas. The result is shown in Fig.1(b) where the white pixels represent the flooded zones while the black ones show non-flooded areas. It was possible to detect the local target (red rectangle in Fig.1(b)) by comparing the segmented image and the HR SAR image; finally, we retrieved the water level. In Fig. 2 the tridimensional model of the local target is reported while in Fig.3 an aerial optical image of the flooded building is shown. The different scattering contributions for the geometric model at issue are distributed on the SAR image, Fig.4. According to [1], from left to right we expect to find: layover area, double reflection line, scattering from the roof and the shadowing. 


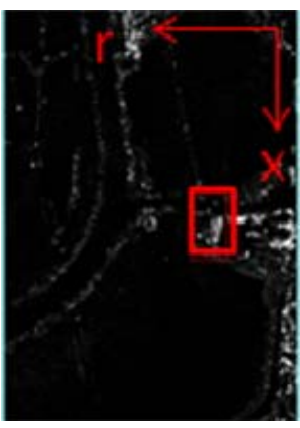

(a)

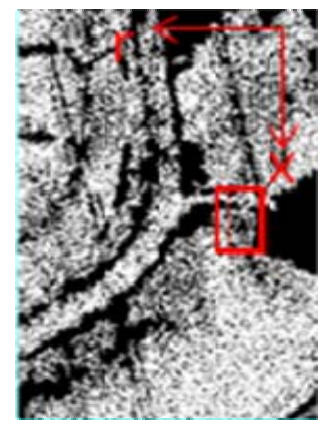

(b)
Fig.1. Region of interest (red rectangle) including the local target taken in the HR SAR image (a) and in the segmented image (b).

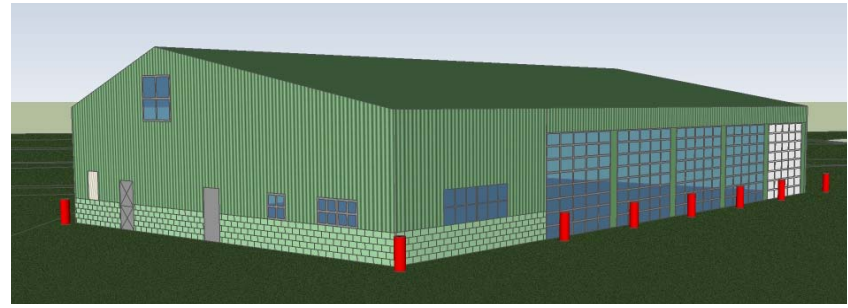

Fig.2. Tridimensional view of the local target.

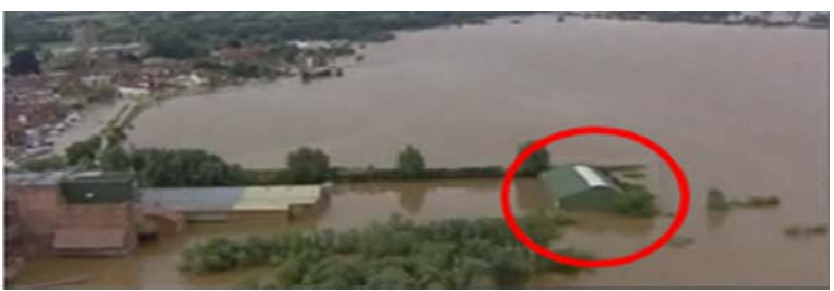

Fig.3. Aerial picture of the local target after flooding. Courtesy of BBC News.

The knowledge on the relevant distance between these contributions has been used to localize the double reflection line. Geometric parameters and materials relevant to the building have been directly acquired in situ, while the angle between the sensor line of flight and the projection of a wall of the building to the ground has been estimated from the image. The geometric parameters of the local target are reported in Table I. The local target is made of three different materials: glass, aluminum and bricks. The dielectric constants of these materials depend on the working frequency. In $\mathrm{X}$ band $(9.65$ $\mathrm{GHz}$ ) the following values can be considered [6-7]:

$$
\varepsilon_{g}^{\prime}=6.2 \quad \varepsilon_{a}^{\prime}=4.0 \quad \varepsilon_{b}^{\prime}=4.5
$$

where $\varepsilon_{g}^{\prime}, \varepsilon_{a}^{\prime}$ and $\varepsilon_{b}^{\prime}$ are the real part of the dielectric constant of the glass, aluminum and bricks respectively. In the same way the imaginary part of the dielectric constants are:

$$
\varepsilon_{g}^{\prime \prime}=0.037 \quad \varepsilon_{a}^{\prime \prime}=6.6 \cdot 10^{7} \quad \varepsilon_{b}^{\prime \prime}=0.3
$$

In addition we estimated that the local target is made of glass for $47 \%$, aluminum for $50 \%$ and bricks for $3 \%$. Performing a weighted average of the dielectric constants as done in [7],

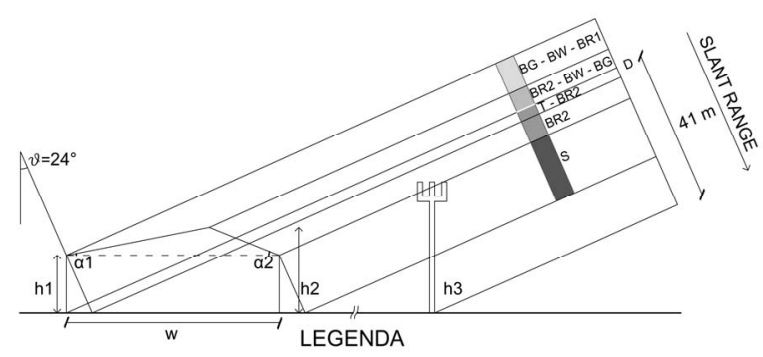

$\mathrm{BW}=$ Backscattering from Wall $\quad \mathrm{BR} 2=$ Backscattering from Roof $2 \quad \mathrm{D}=$ Doublc scattcring $B R 1=$ Backscattering from Roof $1 B G=$ Backscattering from Ground $T=$ Triple scattering

$S=$ Shadow

Fig.4. Cut at constant azimuth of the scene: composition of different contributions of the local target in the SAR image.

TABLE I

\begin{tabular}{|c|c|}
\hline Parameter & Measure \\
\hline Length $l \quad[\mathrm{~m}]$ & 35.3 \\
\hline Width $w \quad[\mathrm{~m}]$ & 20.2 \\
\hline Height $h_{1}[\mathrm{~m}]$ & 5.5 \\
\hline Height $h_{2} \quad[\mathrm{~m}]$ & 8.1 \\
\hline Angle $\alpha_{1}[\mathrm{deg}]$ & 12 \\
\hline Angle $\alpha_{2}$ [deg] & 19 \\
\hline Angle $\varphi[\mathrm{deg}]$ & 12.7 \\
\hline
\end{tabular}

GEOMETRIC PARAMETERS OF THE LOCAL TARGET

it is possible to provide a rough estimate of an equivalent mean relative dielectric constant of the wall:

$$
\varepsilon_{m}=5.1-j 3.3 \cdot 10^{7}
$$

These values are used in the next section for the flood level retrieval.

\section{RESULTS}

In this Section, the proposed algorithm is tested. The ground covered with water is modeled as a Gaussian stochastic process representing the superficial water wavelets due to the wind and rubble. The approach presented in (3) is adopted to evaluate the radar cross section of the double reflection line relevant to the considered building. It gives a closed-form that can be easily inverted. This approximation can be used if $k \cdot \sigma>>1, \sigma$ being the random surface standard deviation. However, the chosen value to describe the stochastic surface does not completely fulfill the previous condition even if $k \cdot \sigma>1$ is satisfied. Thus we still employ GO-GO approximation in according to [8]. In order to enforce the system in (4), two wooden electricity poles are chosen like gauges. In this case the choice is convenient as the electricity poles appear clearly in the image due to the presence of water. The double reflection line of the local target is localized evaluating its distance from a reference electricity pole (Fig.4). Moreover the mean intensity of the double reflection line is estimated with a simple supervised method (Fig.5). 


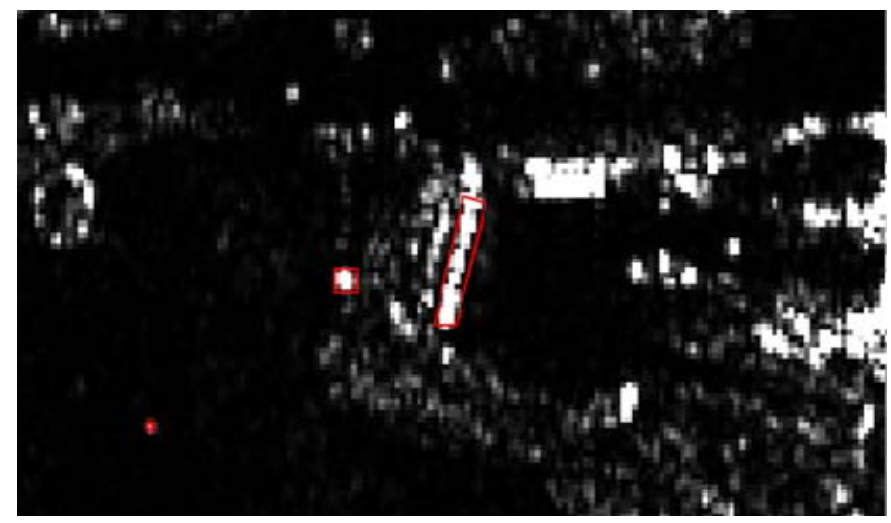

Fig.5. Double reflection lines referring to the local target and two gauge poles.

All the parameters of interest are reported in Table II, where the water dielectric constant is calculated from [9]. The element of scattering matrix $S_{H H}$ has been computed and we found $\left|S_{H H}\right|=1.10707$. Once that all parameters in (3) were known the water level in the surroundings of the local target has been estimated to be $3.17 \mathrm{~m}$ referring to the first equation of (5). It is known that the measured water level at Mythe Bridge water gauge, situated close to the local target, was $12.22 \mathrm{~m}$ a.s. 1 . (above sea level) on the 25 of July 2007 [3]; the water level in ordinary conditions is $8 \mathrm{~m}$ a.s.l., instead. Finally it is possible to calculate the measurement error as:

$$
E=\left(h_{f}-h_{n f}\right)-\hat{h}_{w}=(4.22-3.17) m=1.05 m
$$

where $h_{f}$ and $h_{n f}$ are the water level measured at the water gauge in flood and non-flood conditions respectively while $\hat{h}_{w}$ is the measured water level. The measurement error represents the worst-case because the river embankment has been ignored and so the flooding level is obviously lower than the measured one at water gauge; so we can consider very positively this first experiment result.

\section{CONCLUSIONS}

A local approach for the estimation of flood level from one single SAR image has been here presented. The approach novelty relies on the flooding level being estimated from radiometric parameters of the employed single SAR image. This approach is very useful in case of continuous monitoring of sensible targets such as hospitals, waterworks and power stations that must operate even in emergency situations. We underline that the evaluated error is lower than the spatial resolution and it could be even lower if a Spotlight image were employed as the double reflection line detection would be much more accurate. In order to achieve better results it is necessary to choose gauges in proximity of the local target or to place pole gauges ad hoc. The potential exploitation of this study is huge and this research is timely if we consider the recent increase of flooding events as: Nashville (Tennessee, U.S.A.) in May 2010, China in June 2010, Germany and Poland in August 2010 and India and Pakistan in September
TABLE II

SCENARIO PARAMETERS

\begin{tabular}{|c|c|}
\hline Parameter & Value \\
\hline Dielectric constant of building $\varepsilon_{m}$ & $5.1-j 3.3 \cdot 10^{7}$ \\
\hline Dielectric constant ofwater $\varepsilon_{w}$ & $55-j 38$ \\
\hline Dielectric constant of pole $\varepsilon_{P}$ & $2-j 0.007$ \\
\hline Electricity pole height $h_{A}[\mathrm{~m}]$ & 7.6 \\
\hline Electricity pole height $h_{B}[\mathrm{~m}]$ & 12.5 \\
\hline Building height $h_{C}[\mathrm{~m}]$ & 5.5 \\
\hline Standard deviation $\sigma[\mathrm{m}]$ & 0.01 \\
\hline Correlation length $L[\mathrm{~m}]$ & 0.2 \\
\hline
\end{tabular}

2010. So far the algorithm proposed has been automatized in the classification procedure but the extraction of double reflection lines is still manually performed. The authors are currently working at this task as well as at further scenarios to provide a more reliable performance analysis of their approach.

\section{ACKNOWLEDGMENT}

Part of this work was developed within the project 2202 supported by the Italian Space Agency within the CSK AO and the proposal MTH0183 supported by the German Aerospace Center (DLR) within the TerraSAR-X AO concerning the exploitation of TerraSAR-X data.

\section{REFERENCES}

[1] G. Franceschetti, A. Iodice, D. Riccio, "A Canonical Problem in Electromagnetic Backscattering From Buildings", IEEE Transactions on Geoscience and Remote Sensing, vol. 40, no. 8, August 2002.

[2] R. Guida, A. Iodice, D. Riccio," Height Retrieval of Isolated Buildings From Single High-Resolution SAR Images", IEEE Transactions on Geoscience and Remote Sensing, vol.48, n.7, pp.2967-2979, August 2010.

[3] H. Zwenzner, S. Voigt, "Improved estimation of flood parameters by combining space based SAR data with very high resolution digital elevation data", Hydrol. Earth Syst. Sci., 13, 567-576, 2009.

[4] S. Martinis, A. Twele, S. Voigt, " Towards operational near real-time flood detection using a split-based automatic thresholding procedure on high resolution TerraSAR-X data", Nat. Hazards Earth Syst. Sci., 9, 303-314, 2009.

[5] M. Watanabe, M. Matsumoto, M. Shimada, M. Sato, "GB-SAR/PiSAR simultaneous experiment for a trial of flood area detection", Proceedings of the International Geoscience and Remote Sensing Symposium, Boston (Massachussets,USA), vol.3, pp.III-27-III-30, July $2008 .$.

[6] H. E. Bussey, J. E. Gray, E. C. Bamberger, E. Rushton, G. Russell, B. W. Petley, and D. Morris, "International comparison of dielectric measurements," IEEE Trans. Instrum. Meas., vol. IM-13, no. 4, pp.305311, Aug. 1964.

[7] R. Guida, A. Iodice, D. Riccio, and U. Stilla, "Model-based interpretation of high-resolution SAR images of buildings," IEEE J. Sel. Topics Appl. Earth Obs. Remote Sens., vol. 1, no. 2, pp. 107-119, Jun. 2008.

[8] R.Guida, G.Franceschetti, A.Iodice, D.Riccio, G.Ruello, U.Stilla, "Building Feature Extraction via a Deterministic Approach: Application to Real High Resolution SAR Images", Proceedings of the International Geoscience and Remote Sensing Symposium, pp.2681-2684, Barcelona (Spain), 2007.

[9] F. T. Ulaby, R. K. Moore, A. K. Fung, "Microwave Remote SensingActive and Passive", Volume III. 